\title{
Multidisciplinary evaluation of interstitial lung diseases: current insights
}

\author{
Simon L.F. Walsh \\ Number 1 in the Series "Radiology" \\ Edited by Nicola Sverzellati and Sujal Desai
}

Affiliation: King's College Hospital Foundation Trust, London, UK.

Correspondence: Simon L.F. Walsh, King's College Hospital Foundation Trust, Denmark Hill, London, SE5 9RS, UK. E-mail: slfwalshagmail.com

@ERSpublications

There are uncertainties about the optimal approach to multidisciplinary diagnosis in ILDs and a consensus is needed http://ow.ly/oKtC30blak2

Cite this article as: Walsh SLF. Multidisciplinary evaluation of interstitial lung diseases: current insights. Eur Respir Rev 2017; 26: 170002 [https://doi.org/10.1183/16000617.0002-2017].

ABSTRACT Multidisciplinary team (MDT) diagnosis is regarded as the diagnostic reference standard for interstitial lung disease (ILD). Several studies have reported that MDT diagnosis is associated with higher levels of diagnostic confidence and better interobserver agreement when compared to the individual components of the MDT in isolation. Although this recommendation is widely accepted, no guideline statement specifies what constitutes an MDT meeting and how its participants should govern it. Furthermore, the precise role of an MDT meeting in the setting of ILD may vary from one group to another. For example, in some cases, the meeting will confine its discussion to characterising the disease and formulating diagnosis. In others, management decisions may also be part of the discussion. Surprisingly, there is no consensus on how MDT diagnosis is validated. As multidisciplinary evaluation contains all the available clinical information on an individual patient, there is no reference standard against which the veracity of MDT diagnosis can be tested. Finally, many of these uncertainties surrounding MDT meeting practice are unlikely to be answered by traditional evidence-based studies, which create difficulties when generating guideline recommendations. There is clearly a need for expert consensus on what constitutes acceptable MDT meeting practice. This consensus will need to be flexible to accommodate the variability in resources available to fledgling MDT groups and the variable nature of patients requiring discussion.

\section{Introduction}

Interstitial lung disease (ILD) represents a diverse and challenging group of disorders with varied management approaches and prognoses. In 2002, a joint statement from the American Thoracic Association (ATS) and European Respiratory Society (ERS) on the classification of idiopathic interstitial pneumonia (IIP) recommended an integrated and dynamic approach to the diagnosis of ILD, involving clinicians, radiologists and, when lung biopsy has been performed, pathologists [1]. The 2013 ATS/ERS IIP update statement reinforced this recommendation [2]. In essence, this approach to diagnosis allows the initial impression of the clinician to be modified with the successive integration of multidisciplinary data before the final multidisciplinary team (MDT) diagnosis is rendered. Several studies have reported that

Received: Jan 052017 | Accepted after revision: March 182017

Conflict of interest: None declared.

Provenance: Commissioned article, peer reviewed.

Copyright OERS 2017. ERR articles are open access and distributed under the terms of the Creative Commons Attribution Non-Commercial Licence 4.0. 
MDT diagnosis is associated with higher levels of diagnostic confidence and better interobserver agreement when compared to the individual components of the MDT in isolation [3-6]. Based on the literature of the past decade, the casual reader might suppose that MDT diagnosis is the result of a tripartite discussion between a clinician, a radiologist and a pathologist, each with specialist expertise in ILD, contributing equally to the diagnostic process and meeting in one location. It might be surprising then to find that despite the primacy of MDT diagnosis, no guideline statement describes in detail how a multidisciplinary meeting should be conducted. Many of the specifics remain unclear. Do all cases of ILD require multidisciplinary characterisation? Who are the requisite attendees? How often should an MDT meet? Must all participants be physically present at the meeting or is telemedicine an acceptable form of MDT practice? How is the MDT meeting governed? Is the MDT meeting about formulating diagnosis or does management also require discussion by the group? How is MDT diagnosis validated? Although experts may have well-formed views on all of these issues, fledgling MDT groups may feel disincentivised by these uncertainties. Notwithstanding these unanswered questions, MDT evaluation of ILD has become enshrined in the literature as the optimum approach to diagnostic synthesis. These issues, as they relate to MDT assessment of ILD, will form the focus of this article.

\section{Multidisciplinary evaluation of ILD}

Before the publication of the 2002 ATS/ERS IIP classification, histopathological evaluation was regarded as the gold standard for diagnosis in the setting of diffuse lung disease [7-9]. This widely held belief was reflected in two key ways: 1) the classification of the IIPs was based purely on histopathological appearances, and 2) in several studies, histopathology was the diagnostic reference standard against which the veracity of high-resolution computed tomography (HRCT) was gauged [10-15]. At the turn of the century, three key observations challenged this view and were responsible for a shift in diagnostic thinking, which in turn had a major impact on the role of HRCT within the multidisciplinary framework.

The first critical observation was that interobserver agreement between expert thoracic histopathologists is surprisingly poor [16]. In a study of 133 lung biopsies taken from 83 patients with various diffuse lung diseases, the interobserver agreement among 10 thoracic histopathologists for first-choice diagnoses was classified as poor (0.38 kappa $(\kappa)$ coefficient of agreement). In addition, diagnoses made with $100 \%$ confidence were achieved in only $39 \%$ of cases, with $18 \%$ of first-choice diagnoses being made with low confidence. In this particular study, more than $50 \%$ of the interobserver variation related to the diagnosis of nonspecific interstitial pneumonia (NSIP) and much of the associated diagnostic uncertainty arose from its distinction from usual interstitial pneumonia (UIP) [16]. These results are in contrast to a similar study performed at the same institution, this time quantifying the interobserver agreement for a HRCT diagnosis [5]. In this study, interobserver agreement was moderate to very good (weighted kappa coefficient $\left(\kappa_{\mathrm{w}}\right)$ 0.49-0.70) for a confident HRCT diagnosis in a variety of ILDs. Although a confident diagnosis and an accurate one are not synonymous, several studies have reported that a confident HRCT diagnosis is often correct.

The second issue is that when two or more biopsies are taken from the same patient, divergent histopathological diagnoses are frequent; in other words, surgical lung biopsy (SLB) is liable to "sampling error" $[17,18]$. In the setting of UIP and NSIP, FLAHERTY et al. [17] demonstrated that $26 \%$ of patients had different histopathological diagnoses assigned to different lobes and that, in these patients, the presence of UIP in any sample conferred a poor prognosis. In practical terms, this means that a histopathological diagnosis of NSIP based on a single lung biopsy specimen from one site can be inaccurate.

Finally, histopathological diagnosis is not immutable, even when made by experts. Frequently, histopathological appearances are intermediate between two disease entities and the diagnosis of one entity over another may be a matter of fine judgement (one reason for the poor interobserver agreement reported). It is in these situations that clinical and HRCT data are likely to be most influential. This point is highlighted by the observation that histopathologists will modify or completely change their diagnosis almost $20 \%$ of the time when subsequently provided with clinical and HRCT data [3].

The 2002 ATS/ERS IIP classification statement was the first guideline document to emphasise the importance of an integrated and dynamic interaction between radiologist and histopathologist for diagnostic synthesis [1]. The 2013 ATS/ERS IIP update statement reinforced this approach [2]. In this scheme, a provisional diagnosis may undergo several refinements with the successive integration of multidisciplinary data before the final MDT diagnosis is rendered. This diagnostic approach has been validated by several studies. FLAHERTY et al. [3] examined the cumulative impact of clinical, radiological and histopathological data on diagnostic confidence and interobserver agreement in suspected idiopathic pulmonary fibrosis (IPF). In this study, a diagnosis of IPF made either by a clinician or radiologist, when presentation was considered typical, was accurate in more than $95 \%$ of cases. This is consistent with previous reports in diagnostic studies of diffuse lung disease and was an important step in validating 
non-histopathological diagnosis of IPF based on typical HRCT findings. This study argued against the assignment of a diagnostic gold standard, instead emphasising the "silver standards" of clinical, radiological and pathological data. FLAHERTY et al. [3] investigated how diagnoses changed from the initial individual clinical or HRCT diagnosis to the final clinical-radiological-histopathological diagnosis at MDT discussion. Most significantly, in 19\% of cases, pathologists changed their original diagnosis once the clinical and radiological data became available. Furthermore, agreement between all participants increased when diagnoses were made in an MDT setting.

The benefits of integrating multidisciplinary data when making a diagnosis of diffuse lung disease have been reported in several other studies [4-6]. Specifically from the radiologist's perspective, HRCT may have a significant impact on the final diagnosis, even when a histopathological diagnosis has been made. AzIz et al. [5] have reported on the effect of HRCT data on clinical diagnosis in 168 patients with suspected ILD. In that study, the clinician's first-choice diagnosis changed in 51\% of cases, and diagnostic confidence improved (reflected in a $\kappa_{\mathrm{w}}$ of interobserver agreement between clinicians increasing from 0.47 to 0.72 ) once HRCT data were made available to them. In addition, post-HRCT, the number of differential diagnoses given by all clinicians in the study fell, which suggests that diagnostic precision was improved. HRCT also has an important role in the diagnosis of idiopathic NSIP. In 105 patients with biopsy-proven NSIP according to the 2002 ATS/ERS guidelines, TRAVIS et al. [19] evaluated the impact of clinical and HRCT data on final diagnosis. In this study, 21 cases had their histopathological diagnosis modified by the addition of HRCT data, and another 14 cases were assigned a different diagnosis based on HRCT and clinical data. ThomeER et al. [4] evaluated interobserver agreement between MDTs for the diagnosis of IPF. In this study, although the level of agreement between the radiologists for a radiological diagnosis of UIP was only moderate $\left(\kappa_{\mathrm{w}}=0.40\right)$ and the level of agreement between pathologists for a histopathological diagnosis of UIP was fair $\left(\kappa_{\mathrm{w}}=0.30\right)$, the overall accuracy of the MDT diagnosis of IPF was good $(87.2 \%)$.

A limitation of these studies is that they pre-date the 2011 joint ATS/ERS/Latin American Thoracic Association (ALAT)/Japanese Respiratory Society (JRS) guidelines for the management and diagnosis of IPF as well as the availability of novel antifibrotic IPF drugs, all of which may influence diagnostic decisions [20-22]. A recent international study involving seven expert MDTs demonstrated high levels of inter-MDT meeting agreement for a diagnosis of IPF $(\kappa=0.60)$ and for the diagnostic likelihood of IPF $(\kappa=0.71)$ [6]. Inter-MDT meeting agreement for a diagnosis of connective tissue disease (CTD)-related ILD was also good $(\kappa=0.64)$, while agreement between MDT meetings was poor for idiopathic NSIP $(\kappa=0.25)$ and hypersensitivity pneumonitis $(\kappa=0.24)$. Furthermore, in the specific case of IPF, MDT meetings more frequently assigned high-confidence diagnoses, with higher prognostic significance than the individual components of the MDT meeting (i.e. clinicians, radiologists or pathologists) in isolation.

\section{Unanswered questions}

Although there is a substantial body of literature reporting on the benefits of MDT meetings for diagnosis in the setting of ILD, considerable debate remains on what constitutes best MDT practice or, indeed, if a single ideal MDT model exists [1, 2]. Guideline recommendations leave unanswered many practical questions related to MDT meeting practice. For example, what is the purpose of MDT evaluation of ILD? Who are the requisite participants? How often should an MDT meet? Do all cases of ILD require MDT characterisation? How is an MDT meeting governed? It is noteworthy that, despite many years of research highlighting the benefits of multidisciplinary ILD diagnosis, the fundamental question of how the accuracy of multidisciplinary diagnosis is validated has gone unanswered. Perhaps one reason for this is the disinclination to make guideline statements unless there is an evidence-based answer. However, many questions related to the best practice of multidisciplinary ILD diagnosis cannot be answered using evidence-based methodologies. What follows are a series of questions related to MDT practice with suggested answers based on a combination of evidence and experience. A series of recommendations for MDT meeting practice are provided in table 1.

What is the purpose of MDT evaluation of ILD?

Much of the debate surrounding MDT evaluation of ILD is fuelled by a failure to clarify what its precise role is. In oncology, MDT discussion is an essential component of the patient management pathway. In many cases, a diagnosis has already been established, invariably with histological corroboration, and the focus of discussion is on the treatment of disease and addressing the individual needs of the patient. In contrast, MDT evaluation of ILD is almost always about disease characterisation and formulating a diagnosis. This is rarely a purely evidence-based exercise because the available clinical data on individual patients are highly variable, often making it difficult to apply standardised diagnostic algorithms. Although management recommendations may naturally flow from diagnosis (for example, in IPF, the prescription of antifibrotic therapy is a natural extension of the diagnostic process), any move to require that management decisions must be made by means of MDT discussion should be resisted. Patient frailty and the patient's 


\section{TABLE 1 An approach to multidisciplinary team (MDT) meeting practice}

What is a diffuse lung disease MDT meeting?

What is the main goal of the MDT meeting in the setting of diffuse lung disease?

Who are the requisite participants in the MDT meeting?

Who leads the MDT meeting?

What is the minimum dataset needed for diagnostic synthesis in an individual diffuse lung disease patient? Should the MDT meeting outcome be documented?

Do all cases of DPLD require MDT meeting evaluation?

How often should an MDT meeting take place?

How can DPLD MDT meeting performance be appropriately evaluated?
A forum that enables the integration of all available clinical, radiological and pathological data for an individual patient for the purposes of diagnostic synthesis.

The primary goal of MDT meetings is to discuss and establish diagnosis. In some cases, management strategy inevitably flows from diagnosis, e.g. the prescription of antifibrotic therapy in patients with IPF.

If the primary goal of MDT meetings in the setting of DPLD is diagnostic synthesis, then the requisite participants are the respiratory physician, radiologist and pathologist.

In most cases, the participant who has performed a face-to-face evaluation of the patient should lead the MDT discussion.

The lack of standardised diagnostic criteria in diffuse lung disease means that defining the minimum required dataset is not possible at present.

Ideally, the outcome of the MDT discussion should be recorded in the patient's clinical records. A dedicated proforma for recording MDT discussion outcome may inform service improvements as well as facilitate research.

Experienced MDTs may wish only to discuss cases where there is inter-specialty diagnostic disagreement or where the disease is not easily classified. Less experienced MDTs may initially wish to discuss all cases of diffuse lung disease.

The DPLD MDT meeting frequency will depend on the experience of the group and the number of patients being evaluated. New groups may wish to discuss all cases but may have insufficient patient numbers to fill a weekly MDT meeting. Experienced groups may have large numbers of patient referrals and may need to triage patient that require discussion at a weekly meeting.

MDT meeting performance can be evaluated in terms of diagnostic accuracy, participant experience and cost effectiveness. Diagnostic agreement and diagnostic confidence may be used as surrogates for diagnostic accuracy. Specifically in IPF, outcome distinctions between IPF and other diffuse lung diseases may be used to validate diagnostic accuracy. Participant experience may be assessed using questionnaires and cost-effectiveness studies may be used to assess feasibility.

IPF: idiopathic pulmonary fibrosis; DPLD: diffuse parenchymal lung disease.

own wishes may impact these decisions and therefore are more appropriately taken by an MDT participant who has actually met the patient; in most cases, this is the attendant clinician.

A related issue is whether all cases of suspected ILD require MDT characterisation. This might be dictated by the experience of the MDT group. New groups formed by less experienced participants, dealing with smaller patient cohorts, might be advised to evaluate most if not all patients with suspected ILD. Aside from the benefits to the patient, this approach is likely to broaden the MDT group's experience and establish an ethos of debate and logical thinking. One of the great benefits of MDT evaluation in this setting is that participants are required to defend their opinions and diagnoses in public. However, an experienced and busy group cannot hope to do this. In these circumstances, cases brought to an MDT discussion should be those that are, in the view of the clinician (and possibly the radiologist), in need of more detailed characterisation. A simple example will highlight this point; no amount of MDT discussion will alter a clinician's impression of IPF in an elderly male patient with rapidly progressive dyspnoea, a typical UIP pattern on HRCT, and no identifiable trigger. However, the same radiological presentation in a young female patient with equivocal serology suggesting, but not confirming, the presence of a CTD is likely to require discussion. Patients in whom cryobiopsy or surgical biopsy is being considered almost always require MDT discussion. It should be highlighted that although there are no specific guidelines that state which patients require MDT discussion, prescribing mandatory discussion of all cases of suspected ILD, and in particular IPF, should be avoided.

Lastly, in addition to evaluating new cases, revisiting and revising diagnoses based upon disease behaviour and response to therapy is an important role of MDT discussion. This is of particular importance in patients whose disease behaviour is unexpected and could not have been predicted on initial assessment. This will necessarily include reassessment of lung function and imaging to plot change, and integrating this change into MDT diagnosis. 
Who are the requisite participants in MDT evaluation of ILD?

Based on the original studies by FLAHERTY et al. [3], it might be supposed that multidisciplinary evaluation should at a minimum involve a clinician, a radiologist and a pathologist. In recent times, more expansive models including rheumatologists, thoracic surgeons or ILD nurses have been suggested [23-25], raising the question "Who are the requisite participants in a multidisciplinary evaluation of diffuse lung disease?" As previously discussed, for many MDT groups, the primary goal is establishing a diagnosis rather than formulating a management strategy. This distinction is important: it has a profound impact on the nature of MDT discussion and, more particularly, who must be present. If MDT evaluation in ILD is accepted as being primarily about diagnosis, then the participation of surgeons or specialist nurses is unlikely to have a meaningful impact. Also, those that argue for the compulsory attendance of all manner of people risk discouraging ILD groups who do not have access to these peripheral specialties.

Specifically, the role of the rheumatologist within ILD MDT meetings warrants further discussion. A recent study reported that among seven international expert multidisciplinary groups, new diagnoses of CTD-ILD were constructed in approximately $10 \%$ of patients [6]. On the face of it, one might conclude from this that formal rheumatology participation should be mandatory whenever an MDT diagnosis of ILD is made. A particular challenge faced by MDT groups is separating patients with IIP from those with CTD-related ILD: some patients present with subtle clinical features or serological abnormalities that suggest an autoimmune process but do not meet established criteria for a specific CTD. Recently, an ERS/ ATS task force was formed in order to establish consensus on how to classify these patients (now designated interstitial pneumonia with autoimmune features (IPAF)) and a set of diagnostic criteria have been suggested [26]. These criteria are divided into three domains: a clinical domain, a morphological domain and a serological domain. In the context of these three domains, the importance of rheumatology input lies in the physical examination of the patient, searching for signs of an autoimmune process. For example, distinguishing between an osteoarthritic or inflammatory arthritis might occasionally be a matter of fine judgement, beyond the clinical skills of a pulmonologist. Insisting that a rheumatologist be present at MDT discussions without the benefit of consultation with the patient in person is unlikely to add much of value to the MDT process. Conversely, the integration of lung function, imaging and histological data in all cases is best performed by the ILD physician. The rheumatologist and ILD physician thus play separate roles, which are complementary but mutually exclusive. Clearly, evaluation of patients with suspected autoimmune features by a rheumatologist and ILD physician within a parallel clinic is desirable, but for practical reasons may not always be possible.

A reasonable conclusion, based on a combination of evidence and, most importantly, common sense, is that only a clinician, a radiologist and (in selected cases where biopsy has been performed) a pathologist are mandatory MDT meeting attendees. An interesting related point is how transbronchial cryobiopsy and its impact on the availability of lung biopsy specimens, as well as the rapidly progressing field of molecular biomarkers, might influence who is required to be present at ILD MDT discussions.

\section{How is MDT meeting diagnosis validated?}

Although MDT diagnosis may be associated with superior levels of diagnostic agreement, this does not necessarily indicate more accurate diagnoses. It should be remembered that, although MDT discussion may result in more confident diagnoses and higher levels of diagnostic agreement, the performance of the MDT is intrinsically dependent on the experience of the individual participants. No amount of MDT discussion will overcome the shortcomings of participant inexperience. This raises the question "How is MDT evaluation validated for diagnostic accuracy?" Validation of any diagnostic test is generally an evidence-based process that ensures that the test complies with some accepted reference standard. However, the process of multidisciplinary evaluation involves integrating all available clinical, radiological and pathological data at presentation, which means there is no reference standard against which the veracity of MDT diagnosis can be tested. All data are contained within the MDT diagnosis. Specifically in the case of IPF, one logical approach is to validate MDT diagnosis at presentation against outcome distinctions between IPF and other diffuse lung diseases. In the study by WALSH et al. [6], the accuracy of IPF diagnosis rendered by clinicians, radiologists, histopathologists and the MDT meeting itself were validated against mortality. In this study, trends toward greater prognostic separation of patients based upon an IPF diagnosis made by MDT meetings compared to clinicians, radiologists or pathologists in isolation, supports the current guideline recommendation that MDT evaluation of patients with suspected IPF is the optimal diagnostic approach. Further evaluation of MDT meeting diagnosis in the context of patient outcome is needed.

Where and how often should MDT evaluation of ILD take place?

The frequency of ILD MDT evaluation will often depend on the experience of the group, for several reasons. First, as previous discussed, new groups simply may not have enough cases of suspected ILD to require a weekly meeting. Secondly, it may take time for an MDT group to develop momentum. 
As the service grows, increasing patient numbers and possibly referrals from other centres will mean that full MDT characterisation will be possible only for selected cases. It is at this stage that the MDT group will need to firmly establish its own guidelines for who requires and does not require discussion.

No recommendations on MDT meeting location have been published. Having all participants physically present in one location might be ideal, but not always possible. Some groups may not have local access to expert radiologists or histopathologists, but this expertise may be available through wider networks using telemedicine. In an ongoing study of diagnostic agreement in the setting of ILD, involving 766 pulmonologists, 127 reported having no direct access to ILD imaging expertise, but had indirect access through local networks. This was also true for 195 pulmonologists with respect to the availability of histopathology expertise [27]. Therefore, it may be necessary that future guideline recommendations include telecommunication as an accepted form of multidisciplinary practice (Melissa Wickremasinghe, Imperial College Healthcare NHS Trust, London, UK; personal communication).

\section{MDT governance}

Although several studies have reported on MDT diagnosis regarding diagnostic confidence, interobserver agreement, accuracy and prognostic significance, the more subjective influences on MDT diagnosis, such as participants' personalities, cultural biases or time constraints have not been examined [3-6]. One might be forgiven for assuming, following a review of the current literature, that the clinician, radiologist and pathologist contribute equally and in a consistent fashion between different MDT groups. This is clearly an unrealistic expectation. An important, but difficult to quantify component of MDT meeting dynamics is the effect of individual personalities on the final MDT diagnosis. Guideline descriptions of multidisciplinary diagnosis describe it as a consensus among participants; however, depending on individuals' experience and personality, the final diagnosis may ultimately be more reflective of the strongest voice in the room. Therefore, the question is "Who is responsible for MDT meeting governance and how is this legislated?" In some cases referred for MDT evaluation, a clinician may be seeking radiological corroboration of a diagnosis he or she already has in mind. In this situation, the clinician is likely to lead discussions and formulate the diagnosis. In other cases, the clinician may have a differential diagnosis consisting of two or three possibilities, and requires expert radiological input to refine the diagnosis further. In this situation, the radiologist is likely to have significant impact on the final diagnosis. Lastly, some cases of ILD might be deemed unclassifiable, requiring in-depth MDT discussion and a consensus on the need to perform SLB. Occasionally, in these cases, a single confident MDT diagnosis cannot be achieved.

A recent review of multidisciplinary meeting practices in 12 expert ILD centres by Jo et al. [25] demonstrated significant heterogeneity in meeting format and organisation. This survey reported that the attendant clinician was responsible for leading the meeting $90 \%$ of the time, documenting outcome $70 \%$ of the time, and had the greatest input into formulating diagnosis $60 \%$ of the time. Interestingly, in $30 \%$ of meetings, the final decision on diagnosis was left to the clinician following multidisciplinary discussion.

\section{Multidisciplinary evaluation of suspected IPF \\ Guideline diagnosis versus MDT diagnosis}

One benefit of evidence-based guidelines is that they allow MDT groups with less experience to make diagnoses with high confidence. Application of the 2011 ATS/ERS/ALAT/JRS IPF statement revolves primarily around assigning a CT diagnosis category based on the presence or absence of honeycombing and the exclusion of features considered inconsistent with UIP. In cases where biopsy has been performed, evaluation of the histology follows a similar approach. In recent times, radiological misdiagnosis arising from difficulties applying guideline HRCT diagnosis categories has been the focus of debate. In a survey performed at a Europe-wide IPF meeting, a majority of clinicians stated that they found an HRCT diagnosis of IPF to be reliable from less than one-third of radiologists. Recently, diagnostic agreement among 116 radiologists for the categorisation of 150 cases of fibrotic lung disease based on the 2011 ATS/ ERS/ALAT/JRS HRCT criteria for a UIP pattern was reported as moderate, with no significant improvement among thoracic radiologists with $>25$ years of experience [28]. Given the crucial role that HRCT plays in the initial evaluation of patients with IPF, it might be supposed that radiological misdiagnosis should adversely impact MDT diagnosis. Surprisingly, this is not the case. In contrast to radiological diagnosis of UIP, inter-MDT meeting agreement on the likelihood of IPF is good $\left(\kappa_{\mathrm{w}}=0.71\right)$ [6]. In the case of IPF, experts have taken the requisite time to draw up diagnostic guideline recommendations that can be applied in clinical practice. In sharp contrast, poor levels of inter-MDT agreement have been demonstrated for diseases for which no consensus guideline statements exist, in particular, hypersensitivity pneumonitis and idiopathic NSIP [6].

Despite these benefits, guidelines do not come without difficulties, many of which relate to the "one size fits all" paradigm they attempt to enforce. A prevalent diagnostic problem encountered by MDT groups is 
the IPF patient whose HRCT lacks typical UIP features and is too frail or unwilling to undergo SLB. Currently, in about half of IPF patients, ATS/ERS/ALAT/JRS criteria are not satisfied for IPF and, in these cases, an evidence-based diagnosis is not possible $[29,30]$. Patients find themselves with an unclassifiable condition for which multidisciplinary discussion will result in one of two outcomes: either it is assumed that the diagnosis is not IPF and the clinician will treat the patient accordingly, or a speculative diagnosis of IPF is made and antifibrotic therapy is prescribed. In short, the current diagnostic recommendations for IPF do not cater for a significant number of IPF patients. At the root of this problem is the fact that the $2011 \mathrm{ATS} / \mathrm{ERS} / \mathrm{ALAT} / \mathrm{JRS}$ statement was formulated at a time when no validated IPF therapy existed, but, at the same time, a rigorous diagnostic approach to IPF was needed for use in drug trials. In trials, diagnosis must be standardised to ensure consistency between treated and placebo arms, which in essence means the exclusion of data that are not available in all patients. Most centres do not perform bronchoalveolar lavage in patients with suspected IPF, so the current guideline statement does not emphasise this [20]. Disease behaviour cannot be figured into diagnostic criteria because this information is unavailable at the time of trial enrolment [20]. Current IPF diagnostic guidelines do not incorporate pre-test probability, meaning that strict guideline adherence results in the same diagnostic algorithm being applied to a 35-year-old female patient with a definite UIP pattern on HRCT as would be applied to a 75-year-old man with the same HRCT appearances (assuming no trigger is identified in either case) [20]. Multidisciplinary diagnosis of IPF described in the 2011 ATS/ERS/ALAT/JRS statement is largely a two-dimensional process that combines a histopathological grade and a HRCT classification into a diagnostic probability of IPF represented by four diagnosis categories: IPF, probable IPF, possible IPF or inconsistent with IPF [20]. The application of subtle clinical interpretation, particularly when the imaging or histology is inconclusive, is not highlighted.

When no validated IPF treatment was available, these shortcomings made little practical difference to patients in whom a guideline-based diagnosis of IPF could not be made. However, with the availability of two licensed antifibrotic therapies, accurate diagnosis of IPF has become critical. When the clinical suspicion is one of IPF and the HRCT shows typical UIP, full multidisciplinary characterisation is unlikely to be needed; however, when the HRCT is not that of typical UIP and the patient is disinclined to undergo lung biopsy, multidisciplinary discussion becomes crucial. Unlike the guideline recommendations, which confine diagnosis to the isolated opinions of a radiologist and a pathologist at a point in time, real-world clinical practice involves integrating a broad range of clinical data, which are likely to vary significantly from patient to patient. In addition, cases discussed at presentation can be revisited after a period of observation or therapy, and so disease behaviour can also be considered.

This tension between diagnostic guidelines and multidisciplinary diagnosis may vary depending on the experience of the multidisciplinary group. Experienced groups may feel comfortable overriding guideline recommendations based on expert clinical judgement, but newer groups may initially rely on them to make confident diagnoses. As a practical example, patients who do not meet guideline criteria for a diagnosis of definite IPF might in some cases be prescribed antifibrotic therapy on the basis of a "working diagnosis" of IPF by experienced multidisciplinary groups. In time, this flexible IPF diagnosis, which allows for the variability of real-world clinical practice and incorporates clinical interpretation, will be examined in an evidence-based way and endorsed in future guideline statements.

\section{Emerging diagnostic tools Cryobiopsy}

Based on epidemiological studies from Italy, Belgium, Greece, Spain, the USA and Finland, 28-38\% of patients with IPF were diagnosed by SLB [31-36]. In clinical trials, where the diagnostic approach must be standardised using current guidelines, this proportion increases to as many as $60 \%$ of cases [37-39]. Many patients with suspected IPF may be unable or unwilling to undergo SLB and, at the same time, require histopathological confirmation of UIP to secure a guideline-based diagnosis of IPF. Recent studies suggest that bronchoscopic lung cryobiopsy (BLC) may be a less invasive alternative to SLB. BLC provides large biopsy specimens, is well tolerated by patients, and results in high-confidence diagnoses of UIP in about $50 \%$ of cases [40-46]. In a recent study that evaluated the impact of BLC on MDT diagnosis of IPF, the proportion of high-confidence IPF diagnoses increased from $16 \%$ to $63 \%$ following integration of BLC data into multidisciplinary discussions [47]. Also, BLC changed the initial clinical-radiological impression in $26 \%$ of cases, reclassifying $73 \%$ as IPF. Although histopathology is unlikely to resume its role as a diagnostic gold standard in ILD, BLC, if widely adopted, may have a significant impact on MDT practice in the future.

\section{Biomarkers}

In recent years, biomarker research in ILD has focused on IPF, usually involving relatively small cohorts and without validation. Biomarkers may have several roles, including improving diagnostic accuracy, facilitating management decisions or informing prognosis [48-52]. Rapid advances in our understanding 
of IPF biology have provided a tantalising opportunity for characterising the molecular phenotypes of different ILDs [50, 51, 53-56]. At present, genomics is a highly specialised field, but in time it may become a mainstream form of multidisciplinary data requiring expert interpretation. For this reason, specialists in genetics and molecular biology may become a necessary addition to the multidisciplinary groups of the future.

\section{Conclusion}

Multidisciplinary evaluation is the current diagnostic reference standard for ILD and is reported to improve diagnostic confidence and agreement compared to individual participants of the MDT in isolation. Despite this recommendation being ubiquitous in the ILD literature, many questions regarding the practicalities of MDT meetings remain unclear. Who must attend MDT meetings? Which patients require MDT characterisation and which patients do not? Who is responsible for MDT meeting governance and how is this legislated? An added difficulty is that the answers to many of these questions are likely to come from experience and common sense rather than traditional evidence-based methodologies. Also, the significant variability in available resources, as well as patients requiring MDT evaluation, means that a standardised approach to MDT meetings may not be possible. Although experts will have well-formed views on what represents the ideal MDT meeting, these unanswered questions and the resulting uncertainties may discourage new MDT groups. Appropriate guideline statements will need to combine expert experience with properly designed studies to address these governance-related issues.

\section{Acknowledgements}

The author would like to thank Melissa Wickremasinghe (Trust Lead for Interstitial Lung diseases and Sarcoidosis, Imperial College Healthcare NHS Trust, London, UK), who provided an interview regarding multidisciplinary team meetings.

\section{References}

1 American Thoracic Society, European Respiratory Society. American Thoracic Society/European Respiratory Society international multidisciplinary consensus classification of the idiopathic interstitial pneumonias. Am J Respir Crit Care Med 2002; 165: 277-304.

2 Travis WD, Costabel U, Hansell DM, et al. An official American Thoracic Society/European Respiratory Society statement: update of the international multidisciplinary classification of the idiopathic interstitial pneumonias. Am J Respir Crit Care Med 2013; 188: 733-748.

3 Flaherty KR, King TE Jr, Raghu G, et al. Idiopathic interstitial pneumonia: what is the effect of a multidisciplinary approach to diagnosis? Am J Respir Crit Care Med 2004; 170: 904-910.

4 Thomeer M, Demedts M, Behr J, et al. Multidisciplinary interobserver agreement in the diagnosis of idiopathic pulmonary fibrosis. Eur Respir J 2008; 31: 585-591.

5 Aziz ZA, Wells AU, Hansell DM, et al. HRCT diagnosis of diffuse parenchymal lung disease: inter-observer variation. Thorax 2004; 59: 506-511.

6 Walsh SL, Wells AU, Desai SR, et al. Multicentre evaluation of multidisciplinary team meeting agreement on diagnosis in diffuse parenchymal lung disease: a case-cohort study. Lancet Respir Med 2016; 4: 557-565.

7 American Thoracic Society. Idiopathic pulmonary fibrosis: diagnosis and treatment. International consensus statement. American Thoracic Society (ATS), and the European Respiratory Society (ERS). Am J Respir Crit Care Med 2000; 161: 646-664.

8 Hunninghake GW, Zimmerman MB, Schwartz DA, et al. Utility of a lung biopsy for the diagnosis of idiopathic pulmonary fibrosis. Am J Respir Crit Care Med 2001; 164: 193-196.

9 Katzenstein AL, Myers JL. Idiopathic pulmonary fibrosis: clinical relevance of pathologic classification. Am J Respir Crit Care Med 1998; 157: 1301-1315.

10 Liebow AA. Definition and classification of interstitial pneumonias in human pathology. Prog Respir Res 1977; 8: $1-33$.

11 Liebow AA. The Interstitial Pneumonias. New York, Grune and Stratton, 1969.

12 Mathieson JR, Mayo JR, Staples CA, et al. Chronic diffuse infiltrative lung disease: comparison of diagnostic accuracy of CT and chest radiography. Radiology 1989; 171: 111-116.

13 Grenier P, Valeyre D, Cluzel P, et al. Chronic diffuse interstitial lung disease: diagnostic value of chest radiography and high-resolution CT. Radiology 1991; 179: 123-132.

14 Padley SP, Hansell DM, Flower CD, et al. Comparative accuracy of high resolution computed tomography and chest radiography in the diagnosis of chronic diffuse infiltrative lung disease. Clin Radiol 1991; 44: 222-226.

15 Nishimura K, Izumi $T$, Kitaichi $M$, et al. The diagnostic accuracy of high-resolution computed tomography in diffuse infiltrative lung diseases. Chest 1993; 104: 1149-1155.

16 Nicholson AG, Addis BJ, Bharucha $\mathrm{H}$, et al. Inter-observer variation between pathologists in diffuse parenchymal lung disease. Thorax 2004; 59: 500-505.

17 Flaherty KR, Travis WD, Colby TV, et al. Histopathologic variability in usual and nonspecific interstitial pneumonias. Am J Respir Crit Care Med 2001; 164: 1722-1727.

18 Monaghan $\mathrm{H}$, Wells AU, Colby TV, et al. Prognostic implications of histologic patterns in multiple surgical lung biopsies from patients with idiopathic interstitial pneumonias. Chest 2004; 125: 522-526.

19 Travis WD, Hunninghake G, King TE Jr, et al. Idiopathic nonspecific interstitial pneumonia: report of an American Thoracic Society project. Am J Respir Crit Care Med 2008; 177: 1338-1347.

20 Raghu G, Collard HR, Egan JJ, et al. An official ATS/ERS/JRS/ALAT statement: idiopathic pulmonary fibrosis: evidence-based guidelines for diagnosis and management. Am J Respir Crit Care Med 2011; 183: 788-824. 
21 Richeldi L, du Bois RM, Raghu G, et al. Efficacy and safety of nintedanib in idiopathic pulmonary fibrosis. $N$ Engl J Med 2014; 370: 2071-2082.

22 King TE Jr, Bradford WZ, Castro-Bernardini S, et al. A phase 3 trial of pirfenidone in patients with idiopathic pulmonary fibrosis. N Engl J Med 2014; 370: 2083-2092.

23 National Institute for Health and Care Excellence. Idiopathic Pulmonary Fibrosis in Adults: Diagnosis and Management. Available from www.nice.org.uk/Guidance/CG163. Date last updated: June 2013. Date last accessed: 2017.

24 Tomassetti S, Piciucchi S, Tantalocco P, et al. The multidisciplinary approach in the diagnosis of idiopathic pulmonary fibrosis: a patient case-based review. Eur Respir Rev 2015; 24: 69-77.

25 Jo HE, Corte TJ, Moodley Y, et al. Evaluating the interstitial lung disease multidisciplinary meeting: a survey of expert centres. BMC Pulm Med 2016; 16: 22.

26 Fischer A, Antoniou KM, Brown KK, et al. An official European Respiratory Society/American Thoracic Society research statement: interstitial pneumonia with autoimmune features. Eur Respir J 2015; 46: 976-987.

27 The IPF Project. www.theipfproject.com Date last updated: January 1, 2017.

28 Walsh SL, Calandriello L, Sverzellati N, et al. Interobserver agreement for the ATS/ERS/JRS/ALAT criteria for a UIP pattern on CT. Thorax 2016; 71: 45-51.

29 Wells AU. The revised ATS/ERS/JRS/ALAT diagnostic criteria for idiopathic pulmonary fibrosis (IPF) - practical implications. Respir Res 2013; 14: Suppl. 1, S2.

30 Wells AU. Any fool can make a rule and any fool will mind it. BMC Med 2016; 14: 23.

31 Fernandez Perez ER, Daniels CE, Schroeder DR, et al. Incidence, prevalence, and clinical course of idiopathic pulmonary fibrosis: a population-based study. Chest 2010; 137: 129-137.

32 Hodgson U, Laitinen T, Tukiainen P. Nationwide prevalence of sporadic and familial idiopathic pulmonary fibrosis: evidence of founder effect among multiplex families in Finland. Thorax 2002; 57: 338-342.

33 Karakatsani A, Papakosta D, Rapti A, et al. Epidemiology of interstitial lung diseases in Greece. Respir Med 2009; 103: $1122-1129$

34 Thomeer M, Demedts M, Vandeurzen K. Registration of interstitial lung diseases by 20 centres of respiratory medicine in Flanders. Acta Clin Belg 2001; 56: 163-172.

35 Tinelli C, De Silvestri A, Richeldi L, et al. The Italian register for diffuse infiltrative lung disorders (RIPID): a four-year report. Sarcoidosis Vasc Diffuse Lung Dis 2005; 22: Suppl. 1, S4-S8.

36 Xaubet A, Ancochea J, Morell F, et al. Report on the incidence of interstitial lung diseases in Spain. Sarcoidosis Vasc Diffuse Lung Dis 2004; 21: 64-70.

37 Azuma A, Nukiwa T, Tsuboi E, et al. Double-blind, placebo-controlled trial of pirfenidone in patients with idiopathic pulmonary fibrosis. Am J Respir Crit Care Med 2005; 171: 1040-1047.

38 Noble PW, Albera C, Bradford WZ, et al. Pirfenidone in patients with idiopathic pulmonary fibrosis (CAPACITY): two randomised trials. Lancet 2011; 377: 1760-1769.

39 Taniguchi H, Ebina M, Kondoh Y, et al. Pirfenidone in idiopathic pulmonary fibrosis. Eur Respir J 2010; 35: 821-829.

40 Casoni GL, Tomassetti S, Cavazza A, et al. Transbronchial lung cryobiopsy in the diagnosis of fibrotic interstitial lung diseases. PLoS One 2014; 9: e86716.

41 Poletti V, Casoni GL, Gurioli C, et al. Lung cryobiopsies: a paradigm shift in diagnostic bronchoscopy? Respirology 2014; 19: 645-654.

42 Babiak A, Hetzel J, Krishna G, et al. Transbronchial cryobiopsy: a new tool for lung biopsies. Respiration 2009; 78 : 203-208.

43 Hagmeyer L, Theegarten D, Wohlschlager J, et al. The role of transbronchial cryobiopsy and surgical lung biopsy in the diagnostic algorithm of interstitial lung disease. Clin Respir J 2016; 10: 589-595.

44 Poletti V, Benzaquen S. Transbronchial cryobiopsy in diffuse parenchymal lung disease. A new star in the horizon. Sarcoidosis Vasc Diffuse Lung Dis 2014; 31: 178-181.

45 Pajares V, Puzo C, Castillo D, et al. Diagnostic yield of transbronchial cryobiopsy in interstitial lung disease: a randomized trial. Respirology 2014; 19: 900-906.

46 Mikolasch TA, Porter JC. Transbronchial cryobiopsy in the diagnosis of interstitial lung disease: a cool new approach. Respirology 2014; 19: 623-624.

47 Tomassetti S, Wells AU, Costabel U, et al. Bronchoscopic lung cryobiopsy increases diagnostic confidence in the multidisciplinary diagnosis of idiopathic pulmonary fibrosis. Am J Respir Crit Care Med 2016; 193: 745-752.

48 Spagnolo P, Grunewald J, du Bois RM. Genetic determinants of pulmonary fibrosis: evolving concepts. Lancet Respir Med 2014; 2: 416-428.

49 Putman RK, Rosas IO, Hunninghake GM. Genetics and early detection in idiopathic pulmonary fibrosis. Am J Respir Crit Care Med 2014; 189: 770-778.

50 Rosas IO, Richards TJ, Konishi K, et al. MMP1 and MMP7 as potential peripheral blood biomarkers in idiopathic pulmonary fibrosis. PLoS Med 2008; 5: e93.

51 Kropski JA, Pritchett JM, Zoz DF, et al. Extensive phenotyping of individuals at risk for familial interstitial pneumonia reveals clues to the pathogenesis of interstitial lung disease. Am J Respir Crit Care Med 2015; 191: 417-426.

52 Vij R, Noth I. Peripheral blood biomarkers in idiopathic pulmonary fibrosis. Transl Res 2012; 159: 218-227.

53 Selman M, Pardo A, Barrera L, et al. Gene expression profiles distinguish idiopathic pulmonary fibrosis from hypersensitivity pneumonitis. Am J Respir Crit Care Med 2006; 173: 188-198.

54 DePianto DJ, Chandriani S, Abbas AR, et al. Heterogeneous gene expression signatures correspond to distinct lung pathologies and biomarkers of disease severity in idiopathic pulmonary fibrosis. Thorax 2015; 70: 48-56.

55 Meltzer EB, Barry WT, D'Amico TA, et al. Bayesian probit regression model for the diagnosis of pulmonary fibrosis: proof-of-principle. BMC Med Genomics 2011; 4: 70.

56 Bauer Y, Tedrow J, de Bernard S, et al. A novel genomic signature with translational significance for human idiopathic pulmonary fibrosis. Am J Respir Cell Mol Biol 2015; 52: 217-231. 